\title{
Entrepreneurship
}

Jul a Dez $2020-$ v.4 - n.2

ISSN: 2595-4318

This article is also available online at: www.sustenere.co

\section{Gestão da saúde e segurança do trabalho na construção civil}

O setor da construção civil apresenta índices de acidentes de trabalho elevados. As práticas de gestão da segurança e saúde no trabalho através de suas ferramentas gerenciais, podem minimizar os perigos e riscos de acidentes nos canteiros de obras, preservando o maior capital da empresa, a vida humana. Este artigo tem como objetivo verificar o cenário da gestão da segurança e saúde no trabalho nos empreendimentos da construção civil do município de Unaí, suas principais dificuldades, assim como o uso de EPI's nos canteiros de obras. A coleta de dados se deu por meio de observação in loco e entrevistas semiestruturadas com gestores e colaboradores. $O$ estudo mostra que $84 \%$ dos envolvidos nas atividades da construção civil receberam treinamentos sobre uso adequado dos EPIs e orientações sobre os riscos de sua função. Conclui-se que os responsáveis pelos empreendimentos estudados visam apenas atender as normas regulamentadoras e outros requisitos legais pertinentes ao setor da construção, tendo a figura do técnico de segurança apenas no início do empreendimento, para ajudar com a documentação exigida, passível de fiscalização.

Palavras-chave: Sistemas de gestão; Segurança do trabalho; Proteção individual.

\section{Management of health and safety at work in civil construction}

The civil construction sector has high rates of occupational accidents. Occupational health and safety management practices, through their management tools, can minimize the dangers and risks of accidents at construction sites, preserving the company's largest capital, human life. This article aims to verify the scenario of safety and health management at work in civil construction projects in the municipality of Unaí, its main difficulties, as well as the use of PPE's on construction sites. Data collection took place through on-site observation and semi-structured interviews with managers and employees. The study shows that $84 \%$ of those involved in civil construction activities received training on the proper use of EPIs and guidance on the risks of their function. It concluded that those responsible for the studied enterprises aim only to meet the regulatory standards and other legal requirements pertinent to the construction sector, having the figure of the safety technician only at the beginning of the enterprise, to help with the required documentation, subject to inspection.

Keywords: Management systems; Workplace safety; Individual protection.

Topic: Negócios da Construção Civil

Reviewed anonymously in the process of blind peer.
Received: $17 / 03 / 2020$

Approved: 01/04/2020

Marinaldo Loures Ferreira (iD)

Universidade Federal dos Vales do Jequitinhonha e Mucuri, Brasil

http://lattes.cnpq.br/2795031758366655

http://orcid.org/0000-0002-8106-2793

marinaldo79@gmail.com

Referencing this:

FERREIRA, L. M.. Gestão da saúde e segurança do trabalho na construção civil. Entrepreneurship, v.4, n.2, p.43-51, 2020. DOI: http://doi.org/10.6008/CBPC2595-4318.2020.002.0005 


\section{INTRODUÇÃO}

A construção Civil é a uma área com capacidade bem expressiva na economia do pais, possui a capacidade de elevar a taxa de emprego e de renda, assim como o desenvolvimento social. Entende-se como construção civil, o conjunto de atividade, do ramo da engenharia, que tem como finalidade transformar materiais e espaços de acordo com a necessidade do homem, através da execução de projetos previamente elaborados, respeitando os princípios técnicos e as normas vigentes, caracterizando-se como atividades que envolvem a instalação, reparação, equipamentos e edificações (GOMES et al., 2019; OLIVEIRA, 2012).

No Brasil o setor da construção civil teve um crescimento de 1,6\% no ano de 2019 e com perspectiva de crescimento em torno de 3\% no ano de 2020 (CBIC, 2020). Sendo responsável também pelo aumento no número de acidentes de trabalho, no ano de 2018, foram 623.786 acidentes, sendo 2.022 notificações de acidentes de trabalho com óbitos (SMARTLAB, 2020).

Entre as estratégias para a redução de acidentes do trabalho na construção, pode-se citar a adoção de práticas gestão de segurança e saúde do trabalho (SST). A prática de gestão de SST configura no processo gerencial, empregando uma ou mais técnicas e ferramentas, de características obrigatória pela legislação ou voluntária, cujo objetivo principal visa o controle de riscos relacionados à SST (BRIDI et al., 2013).

Estudos apontam que investir SST torna a empresa competitiva e diferenciada, promovendo melhores resultados, equipes com alta performance e imagem positiva perante a sociedade, ademais, implantar programas de prevenção não é custo e sim investimento estrategicamente motivacional aos colabores, influenciando diretamente no processo produtivo (SILVA et al., 2016).

Nesta perspectiva, o presente trabalho tem como objetivo verificar o cenário da gestão da SST nos empreendimentos da construção civil do município de Unaí, suas principais dificuldades, assim como o uso de EPI's nos canteiros de obras. A saúde e a segurança dos trabalhadores são o ponto principal de atenção dentro de uma organização, perdas materiais e humanas abala a credibilidade de investidores, clientes e perante a sociedade (FERREIRA, 2019). Ademais, as pessoas constituem o principal patrimônio das organizações, sendo vital para o sucesso de qualquer empreendimento (CHIAVENATO, 2014).

\section{REVISÃO TEÓRICA}

\section{Sistemas de gestão da segurança e saúde no trabalho}

Um sistema de gestão visa fornecer uma estrutura para gerenciar os riscos e oportunidades de Segurança e Saúde Ocupacional (SSO), prevenindo lesões e problemas de saúde relacionados ao trabalho, proporcionando locais de trabalho seguros e saudáveis, eliminado os perigos e minimizando os riscos através de medidas preventivas e de proteção efetivas (ABNT, 2018).

Reconhecendo a importância do tema e da padronização da gestão, a International Organization for Standardization - ISO lançou a norma ISO 45001:2018 ${ }^{1}$, ampliando o debate sobre a temática e a adoção

\footnotetext{
${ }^{1}$ A ABNT/Editora Publicações lançou a ISO 45001:2018 PT substituindo a BS OHSAS 18001:2007 PT. Sendo disponibilizada na ABNT catalogo. Porém, para fins de avaliação da conformidade cabe salientar que somente o documento original da ISO poderá ser utilizado.
} 
dessas diretrizes pelas empresas de forma global, especificando os requisitos para um sistema de gestão de SST (SGSST) e fornecendo orientações para o seu uso (SEFRIAN, 2019).

De acordo com a ISSO 45001:2018, a organização deve estabelecer, implementar e manter um processo para eliminar as fontes de risco, assim como a redução dos riscos de SST, adotando a seguinte hierarquia de controles: Eliminação da fonte de risco; Substituição do processo por processos, operações, materiais e equipamentos menos perigosos; Adoção de controles de engenharia e reorganização do trabalho; Adoção de controles administrativos, incluindo treinamento; Uso de EPI adequado.

Os SGSST's são ferramentas gerenciais que contribuem para o desempenho das empresas referente às questões de segurança e saúde ocupacional, visa o atendimento às legislações, o aumento da produtividade, a redução de acidentes, a credibilidade perante a opinião pública e promove a conscientização dos envolvidos (OLIVEIRA et al., 2010).

Para a formatação de um SGSST, devem ser levantados e avaliados todos os perigos e riscos relacionados a cada atividade de trabalho, realizando o monitoramento do cumprimento dos deveres das legislações aplicáveis, definindo controles operacionais para mitigação ou eliminação desses perigos e riscos, proporcionando um ambiente cada vez mais seguro e saudável aos colaboradores (SEFRIAN, 2019).

\section{A segurança do trabalho na Construção Civil}

A Segurança do trabalho é definida por normas e leis previstas na Consolidação das Leis do Trabalho (CLT) e regulamentadas pelo Ministério do Trabalho e Emprego (MTE) através de Normas Regulamentadoras (NRs). Essas normas são disponibilizadas no site da Escola Nacional da Inspeção do Trabalho - ENIT² (BRASIL, 2020a) de forma gratuita, sendo composto por trinta e sete documentos, dentre eles estão descritas no quadro 1.

Quadro 1: Trinta e sete normas.

\begin{tabular}{|c|}
\hline eção Prévia (REVOGADA pela PORTARIA SEPRT n. ㅇ 915, de 30 de julho de 2019, publicada no DOU de 31 \\
\hline NR - 3 - Embargo ou Interdição (Última modificação: Portaria SEPRT 1069, de 23/09/2019); \\
\hline $\begin{array}{l}\text { NR - } 4 \text { - Serviços Especializados em Engenharia de Segurança e em Medicina do Trabalho (Última modificação: Portaria MTPS 510, } \\
\text { de 29/04/2016); }\end{array}$ \\
\hline NR - 5 - Comissão Interna de Prevenção de Acidentes (Última modificação: Portaria SEPRT 915, de 30/07/2019); \\
\hline NR - 6 - Equipamentos de Proteção Individual (EPI) (Última modificação: Portaria MTb 877, de 24/10/2018); \\
\hline NR - 7 - Programas de Controle Médico de Saúde Ocupacional (PCMSO) (Última modificação: Portaria MTb 1031, d \\
\hline NR - 8 - Edificações; \\
\hline $\begin{array}{l}\text { NR - } 9 \text { - Programas de Prevenção de Riscos Ambientais (Última modificação: Portarias SEPRT n. o } 1.358 \text { e 1.359, de } 09 \text { de dezembro } \\
\text { de 2019); }\end{array}$ \\
\hline NR - 10 - Segurança em Instalações e Serviços em Eletricidade (Última modificação: Portaria SEPRT 915, de 30/07/2019); \\
\hline NR - 11 - Transporte, Movimentação, Armazenagem e Manuseio de Materiais; \\
\hline NR - 12 - Segurança no Trabalho em Máquinas e Equipamentos (Última modificação: Portaria SEPRT 916, de 30 \\
\hline NR - 13 - Caldeiras, Vasos de Pressão e Tubulações (Última modificação: Portaria SEPRT 915, de 30/0 \\
\hline NR - 14 - Fornos; \\
\hline NR - 15 - Atividades e Operações Insalubres (Última modificação: Portaria SEPRT n. ㅇ 1. \\
\hline NR - 16 - Atividades e Operações Perigosas (Última modificação: Portaria SEPRT n. ํ 1.357, de \\
\hline NR - 17 - Ergonomia (Última modificação: Portaria 876, de 24/10/2018); \\
\hline $\begin{array}{l}\text { VR - } 18 \text { - Condições e Meio Ambiente de Trabalho na Indústria da Construção (Última modificação: P } \\
\text { 10/02/2020); }\end{array}$ \\
\hline
\end{tabular}

2 ENIT - Escola Nacional da Inspeção do Trabalho (2020), vinculada à secretaria de inspeção do trabalho - SIT, órgão destinado a coletar, registrar, produzir e disseminar conhecimento dirigido às atividades da inspeção do trabalho. 


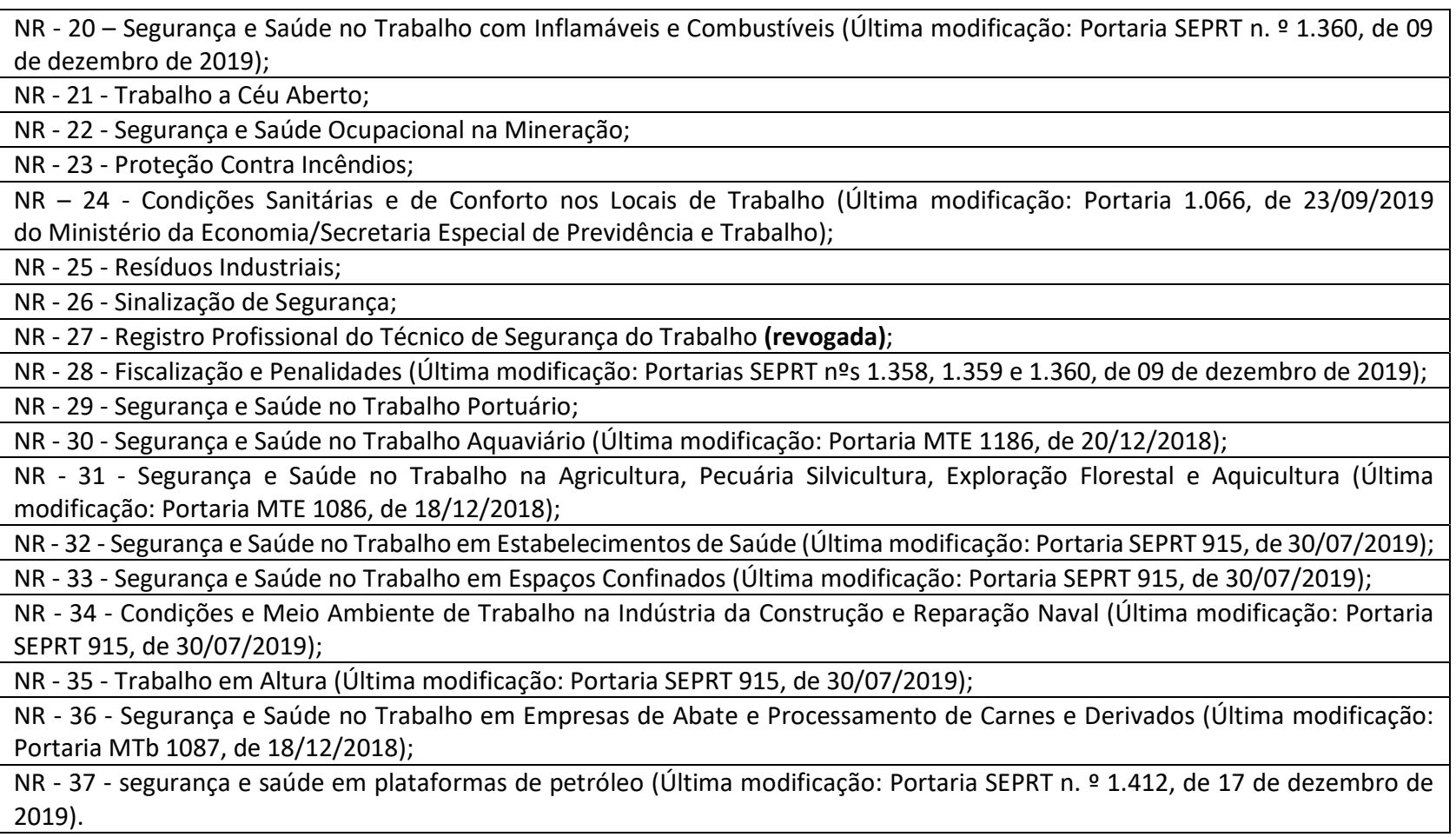

Dada a extinção do Ministério do Trabalho no dia $1^{\circ}$ de janeiro de 2019 , as normas regulamentadoras e demais assuntos referentes à fiscalização do trabalho, assim como outras partes das atribuições desse Ministério, passaram a integrar o Ministério da Economia conforme seção VII, Art. 31 da Lei no 13.844, de 18 de junho de 2019 (BRASIL, 2019a).

Dentre as NRs, aquela que se destina exclusivamente à indústria da construção civil é a NR 18 (BRASIL, 2020b). Portanto, faz-se necessário que sejam conhecidas todas as particularidades trazidas nesta norma. Há outras também não menos importante, que são imprescindíveis na área da construção, dentre elas, a NR 4, $5,6,7$ e 9 .

Há programas relacionados à segurança e à saúde do trabalhador de implementação obrigatória e que, portanto, precisam ser conhecidos pelos profissionais que atuam no setor da construção civil: Programa de Controle Médico de Saúde Ocupacional (PCMSO) - NR 7 (BRASIL, 2018). Dentro das diretrizes do PCMSO, qualquer que seja a metodologia a ser empregada no seu desenvolvimento, ela ocorrerá sob o aspecto individual de cada trabalhador, com um completo acompanhamento desde o seu ingresso na empresa até o seu desligamento (CBIC, 2017).

Dentre as ações realizadas no período de vigência do PCMSO, tem-se a obrigatoriedade da realização, minimamente, dos seguintes exames médicos (BRASIL, 2018): Admissional: A ser realização antes de o funcionário iniciar suas atividades na empresa; Periódico: A ser realizado anualmente para funcionários menores de 18 anos ou maiores de 45 anos e a cada dois anos para os demais funcionários; De retorno ao trabalho: Após afastamento por período igual ou superior a trinta dias em função de parto, doenças ou acidentes (de natureza ocupacional ou não); De mudança de função: A ser realizado antes da data de realização da mudança de função; O Programa de Prevenção de Riscos Ambientais (PPRA) - NR 9, é uma exigência obrigatória a todos os empregadores, independentemente do ramo de atividade e do número de empregados, busca através da antecipação, reconhecimento, avaliação e consequente controle da 
ocorrência de riscos ambientais existentes ou que venham a existir no ambiente de trabalho, tendo em consideração a proteção do meio ambiente e dos recursos naturais (BRASIL, 2019b).

Pela NR-9 os principais agentes de risco a serem considerados no PPRA são os Físicos: ruído, vibrações, pressões anormais, temperaturas extremas (frio e calor), radiações ionizantes e não ionizantes, infrassom e ultrassom; os Químicos: substâncias, compostos e produtos nas formas de poeiras, névoas, fumos, gases ou vapores; e os Biológicos: bactérias, fungos, parasitas, protozoários, vírus, entre outros.

Programa de Condições e Meio Ambiente de Trabalho na Indústria da Construção (PCMAT) - NR 18 (BRASIL, 2020b). O PCMAT deve ser entendido como um projeto de SST específico para um determinado canteiro de obras e não como um manual ou coleção de regras e figuras do tipo "pode", "não pode" para ser apresentado numa possível fiscalização (CBIC, 2017).

O PCMAT consiste em um conjunto de ações e recursos de caráter obrigatório, visando garantir a saúde e a integridade dos trabalhadores da construção civil, prevenindo acidentes do trabalho em canteiro de obras durante todas as fases da construção e estabelecendo condições adequadas de conforto, asseio e higiene ocupacional (SEFRIAN, 2019).

\section{METODOLOGIA}

\section{Método de pesquisa}

A realização deste trabalho se deu por meio de uma pesquisa qualitativa, com base na metodologia de estudo de caso. A pesquisa qualitativa pode e, muitas vezes, necessita ser complementada com recursos de outros tipos de pesquisa como a quantitativa, para atingir os objetivos propostos e apresentação de seus resultados (LARA et al., 2011). Se preocupa fundamentalmente com a compreensão e a interpretação do fenômeno estudado em seus cenários naturais, tendo como principal objetivo compreendê-lo, explorá-lo e especificá-lo (AUGUSTO et al., 2013).

Os instrumentos de coleta de dados basearam-se em entrevistas semiestruturadas com técnicos de segurança, trabalhadores dos canteiros de obras e alguns funcionários-chave dos empreendimentos estudados. Também foram realizadas observações in loco, por meio de visitas com o intuito de observar a postura dos envolvidos no dia a dia em relação à SST.

Portanto, foram analisados nesta pesquisa seis empreendimentos da construção civil, com seus estágios variados, algumas na fase de acabamento e outras na fase de infraestrutura e supra estrutura. Procurou-se, verificar as práticas de gestão da SST nos empreendimentos, assim como o envolvimento dos colabores e as principais dificuldades.

\section{Área de estudo}

O estudo foi realizado no município de Unaí, localizado no Noroeste de Minas Gerais, com $8.448,082 \mathrm{~km}^{2}$ de unidade territorial e população estimada em 2019 de 84.378 habitantes (IBGE, 2019). Se destacando por possuir a maior área equipada por pivôs centrais do país de acordo com levantamento de 
2017, um total de 65.930 hectares, contribuindo de forma significativa para o aumento e a estabilidade da oferta de alimentos no país.

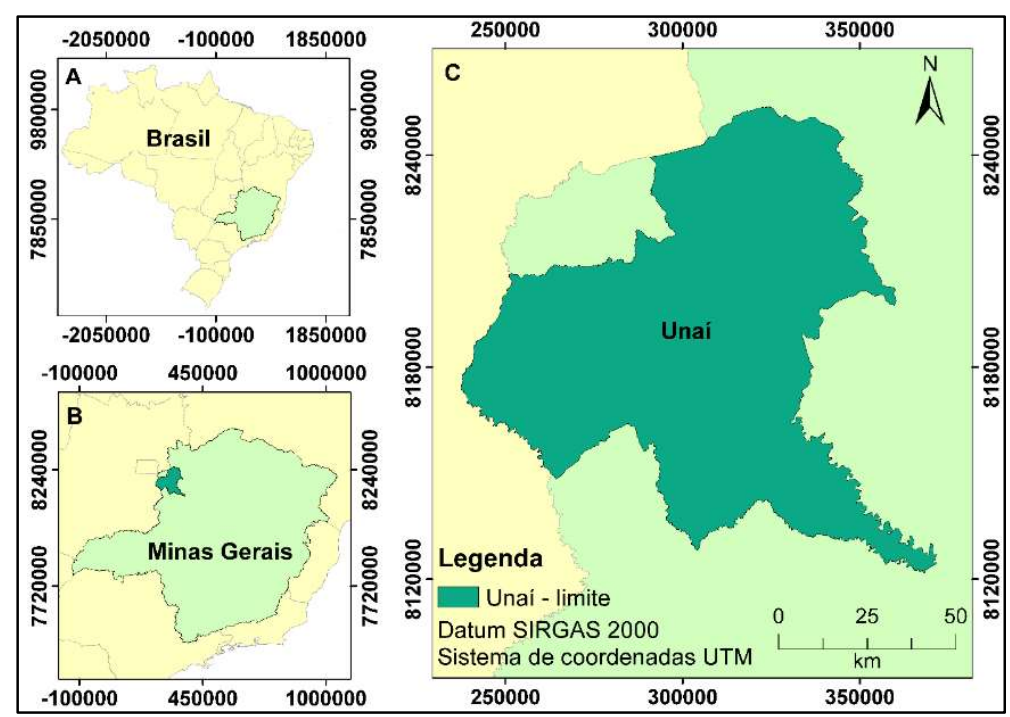

Figura 1: Mapa de localização. A) Localização de Minas Gerais. B) Município de Unaí em Minas Gerais. C) Limite de Unaí.

Ainda segundo informações do (IBGE, 2019) o município figura entre os primeiros colocados no ranking de maior Produto Interno Bruto (PIB) agropecuário de Minas Gerais, refletindo diretamente no setor da construção civil, atividade está, que proporciona um forte impacto no desenvolvimento econômico da cidade.

\section{RESULTADOS E DISCUSSÃO}

Treinamentos e capacitações sobre segurança e saúde do trabalho são ferramentas de SGSST importantes para garantir que os trabalhadores adquiram conhecimento a respeito dos possíveis riscos nas atividades que desenvolverão e adquiram habilidades para evitá-los ou controlá-los (SEFRIAN, 2019).

Nesta perspectiva, o estudo mostra que $84 \%$ dos envolvidos nas atividades da construção civil dos seis empreendimentos avaliados receberam treinamentos sobre uso adequado dos EPIs e orientações sobre os riscos de sua função. Tais treinamentos segundo pesquisa (figura 2), ocorrem ao iniciar o empreendimento, após ser contratado no decorrer das obras e em alguns casos uma vez ao ano. E $16 \%$ afirmam não ter recebido treinamento, fato este, devido a contratação no decorrer da execução do empreendimento, motivado pelo dinamismo das atividades.

Os empreendimentos estudados possuem Grau de Risco 3 de acordo com o disposto no quadro I da NR 4 (BRASIL, 2016), portanto conforme o quadro II da mesma norma, estão dispensados de contratarem um profissional de SST, sendo obrigatório a partir de 100 colaboradores e empresas com GR 4 somente estão obrigadas a terem SESMT a partir de 50 funcionários, exigência esta, que promove a falta de um profissional nos empreendimentos estudados, favorecendo o descompromisso com a SST e tornando a gestão deficiente.

É recomendável que em toda obra, independentemente do seu porte, o engenheiro responsável tenha suporte de um profissional de segurança do trabalho para assessorá-lo, seja como empregado ou como 
prestador de serviço autônomo (CBIC, 2017).

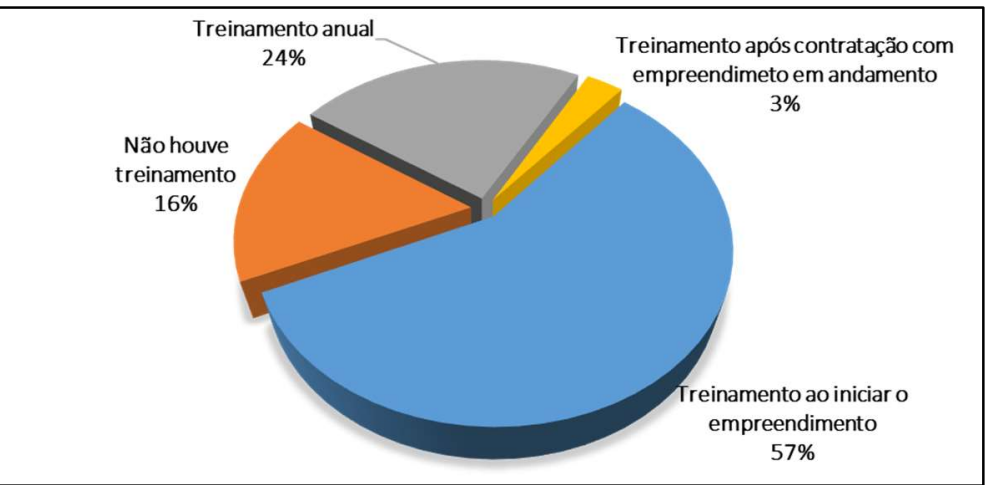

Figura 2: Treinamentos sobre uso de EPIs/orientações sobre risco da função.

Quanto ao fornecimento de EPIs, pôde-se constatar que as empresas exigem e fornecem aos seus colabores. De um modo geral, a maioria dos trabalhadores que atua no canteiro de obras utilizam os EPIs recomendados, exceção daqueles que alegam dificuldades de locomoção quanto ao uso do cinto de segurança.

A deficiência mais visível, observada no estudo, foi relacionado a instalações e Equipamentos de Proteção Coletiva (EPCS), onde todos os empreendimentos estavam em desconformidade com o exigido pelas normas. São exemplos de EPC: o sistema de guarda-corpo e rodapés; plataformas de proteção; sistema limitador de queda em altura (SLQA); tela fachadeira; linha de vida e pontos de ancoragem (SEFRIAN, 2019).

As principais dificuldades referentes a ao gerenciamento do SGSST dos empreendimentos se resumem conforme (quadro 2), onde todas praticamente compartilham das mesmas observações.

Quadro 2: Principais dificuldades encontradas no gerenciamento do SGSST nos empreendimentos de construção civil estudados.

\begin{tabular}{|l|}
\hline CONSTRUÇÃO CIVIL \\
\hline Dificuldade na elaboração de procedimentos e instruções; \\
\hline Falta de profissional específico para gerir os procedimentos; \\
\hline Resistência às mudanças; \\
\hline Baixo índice de escolaridade dos funcionários; \\
\hline Falhas na comunicação interna; \\
\hline Baixo índice de conscientização dos funcionários; \\
\hline Ausência de indicadores de desempenho em segurança e saúde no trabalho; \\
\hline Não estabelecimento da segurança e saúde como um dos objetivos estratégicos da empresa. \\
\hline
\end{tabular}

Um passo importante para facilitar a gestão da SST, seria a realização de Diálogo Diário de Segurança (DDS), recurso este, pouco aplicado nos canteiros de obras. Devido ao andamento do empreendimento e cronograma apertado, segundo a pesquisa 50\% responsáveis aplicam o DDS uma vez por semana e os outros $50 \%$ a cada quinze dia ou quando houver a necessidade. Porém, afirmam que $90 \%$ do momento é para tratar de assuntos referentes a execução das atividades e raramente abordam a segurança do trabalho.

\section{CONCLUSÕES}

Diante do exposto, concluiu-se que os responsáveis pelos empreendimentos estudados têm conhecimento dos riscos relacionados a SST, porém, seus objetivos visam apenas atender as normas regulamentadoras e outros requisitos legais pertinentes ao setor da construção. A figura do técnico de 
segurança se torna usual apenas no início do empreendimento, para ajudar com a documentação exigida, passível de fiscalização, uma vez que, a NR 4 desobriga as empresas de tal profissional em função do grau de risco das atividades relacionada ao número de colaboradores.

Por ser a produção o foco principal, os responsáveis sempre alegam não dispor de recursos para manter um técnico no canteiro, assim como, para correção de situações de risco, ainda que o recurso seja apenas o treinamento e comprometimento com a gestão de SST. Investir em treinamentos técnicos e comportamentais, permite desenvolver competências específicas nos colaboradores, tornando-os mais produtivos, minimizando os riscos de acidentes pela conscientização e conhecimento dos perigos e riscos que envolve as atividades individuais e coletivas dentro dos canteiros de obras.

Quanto aos colaboradores, observa-se uma grande resistência com a SST, argumentam que nunca sofreram acidentes, que são cuidadosos, não havendo a necessidade de usar os equipamentos de proteção individual que são fornecidos pelo empreendimento, assim como, alguns equipamentos de proteção coletiva. Fato este, comum na construção civil em geral, motivado pela baixa escolaridade da maior parte dos colaboradores e falta de fiscalização efetiva por um responsável da SST. E a consequência desta negligencia, é refletida no índice de acidentes do setor, justificando o fato de ser tão expressivo. Propõe-se que a temática do assunto pesquisado seja objeto de posteriores estudos mais aprofundados, associando a gestão com metodologias inovadoras de treinamentos, pois o campo da gestão da SST é amplo, com inúmeras possibilidades de pesquisas.

\section{REFERÊNCIAS}

ABNT. Associação Brasileira de Normas Técnicas. ISO 45001: Sistemas de gestão de saúde e segurança ocupacional: Requisitos com orientação para uso. ABNT, 2018.

AUGUSTO, C. A.; SOUZA, J. P.; DELLAGNELO, E. H. L.; CARIO, S. A. F.. Pesquisa Qualitativa: Rigor metodológico no tratamento da teoria dos custos de transação em artigos apresentados nos congressos da Sober (2007-2011). Revista de Economia e Sociologia Rural, v.51, n.4, p.745-764, 2013. DOI: https://doi.org/10.1590/S0103-20032013000400007

BRASIL. NR 4: Serviços Especializados em Engenharia de Segurança e em Medicina do Trabalho. Portaria MTPS n. ${ }^{\circ}$ 510, de 29 de abril de 2016. Brasília, 2016.

BRASIL. NR 7: Programa de Controle Médico de Saúde Ocupacional. Portaria MTb n. ${ }^{\circ} 1.031$, de 06 de dezembro de 2018. Brasília, 2018.

BRASIL. Lei n. 13.844, de 18 de junho DE 2019. Estabelece a organização básica dos órgãos da Presidência da República e dos Ministérios. Brasília: DOU, 2019a.

BRASIL. NR 9: Programa de Prevenção de Riscos Ambientais. Portaria SEPRT n 1.359, de 09 de dezembro de 2019. Brasília, 2019b.

BRASIL. Escola Nacional da Inspeção do Trabalho. Normas Regulamentadoras. Brasília, 2020a.

BRASIL. NR18: Condições e Meio Ambiente de Trabalho na
Indústria da Construção. Portaria SEPRT n. ${ }^{\circ} 3.733$, de 10 de fevereiro de 2020. Brasília, 2020b.

BRIDI, M. E.; FORMOSO, C. T.; PELLICER, E.; FABRO, F.; CASTELLHO, M. E. V.; ECHEVESTE, M. E. S.. Identificação de práticas de gestão da segurança e saúde no trabalho em obras de construção civil. Ambiente Construído, v.13, n.3, p.43-58, 2013. DOI: http://doi.org/10.1590/S1678$\underline{86212013000300004}$

CBIC. Câmara Brasileira da Indústria da Construção. Guia para gestão de segurança nos canteiros de obra: orientação para prevenção dos acidentes e para o cumprimento das normas de SST. Brasília: CBIC, 2017.

CBIC. Câmara Brasileira da Indústria da Construção. PIB Brasil e Construção Civil. Brasília: CBIC, 2020.

CHIAVENATO, I.. Gestão de pessoas: o novo papel dos recursos humanos nas organizações. 4 ed. Barueri: Manole, 2014.

FERREIRA, M. L.. O coaching como ferramenta para potencializar a gestão do conhecimento na segurança do trabalho. Revista Científica Semana Acadêmica, v.1, 2019.

GOMES, B.; SILVA, D. A.. Construção Civil: Importância do Planejamento de obras Construction: Importance of Construction Planning. Revista Científica Semana Acadêmica, p.1-18, 2019. 
SEFRIAN, H. P.. Segurança e Saúde do Trabalho na Indústria da Construção Civil. São Carlos: Scienza, 2019.

IBGE. Instituto Brasileiro de Geografia e Estatística. IBGE Cidades. Unaí: IBGE, 2020.

LARA, A. M. D. B.; MOLINA, A. A.. Pesquisa Qualitativa: Apontamentos, Conceitos E Tipologias Discutindo a Dicotomia Entre a Pesquisa Qualitativa E a Pesquisa Quantitativa. Maringá: EEduem, 2011.

SMARTLAB. Observatório de Segurança e Saúde no Trabalho. Notificações de Acidentes de Trabalho. SMARTLAB, 2020.
OLIVEIRA, O. J.; OLIVEIRA, A. B.; ALMEIDA, R. A..

Management of occupational health and safety in automotive battery companies: A study to identify best practices. Produção, v.20, n.3, p.481-490, 2010.

OLIVEIRA, V. F.. O papel da indústria civil na organização do espaço e do desenvolvimento regional. In: CONGRESSO INTERNACIONAL DE COOPERAÇÃO UNIVERSIDADEINDÚSTRIA. Anais. Taubate, 2012.

SILVA, K. P.; NASCIMENTO, R. F.; BOTELHO, M. A. S.. Segurança no trabalho: uma ferramenta competitiva. In: SIMPÓSIO DE EXCELÊNCIA EM GESTÃO E TECNOLOGIA, 13. Anais. SEGET, 2016.

A CBPC - Companhia Brasileira de Produção Científica (CNPJ: 11.221.422/0001-03) detém os direitos materiais desta publicação. Os direitos referem-se à publicação do trabalho em qualquer parte do mundo, incluindo os direitos às renovações, expansões e disseminações da contribuição, bem como outros direitos subsidiários. Todos os trabalhos publicados eletronicamente poderão posteriormente ser publicados em coletâneas impressas sob coordenação da Sustenere Publishing, da Companhia Brasileira de Produção Científica e seus parceiros autorizados. Os (as) autores (as) preservam os direitos autorais, mas não têm permissão para a publicação da contribuição em outro meio, impresso ou digital, em português ou em tradução. 